\title{
Modelagem matemática do processo de secagem da massa fibrosa de mandioca
}

\author{
Gabriel L. Castiglioni' ${ }^{1}$, Flávio A. da Silva ${ }^{1}$, Márcio Caliari' ${ }^{1} \&$ Manoel S. Soares Júnior ${ }^{1}$
}

\section{RESUMO}

O presente trabalho teve por objetivo elaborar um modelo matemático para o processo de secagem da massa fibrosa de mandioca e compará-lo com modelos da literatura. O método dos mínimos quadrados foi usado para ajustar os valores experimentais a um modelo semi-empírico. Os resultados experimentais e preditos foram avaliados estatisticamente por análise de variância, para verificar diferenças significativas. As variáveis estudadas foram temperatura e fluxo de ar em secador de bandeja. O modelo construído e o de Page apresentaram valores de erro relativo médio inferiores a $6 \%$, podendo ser utilizados para a predição da cinética de secagem nas condições.

Palavras-chave: Manihot esculenta Crantz, resíduo, subproduto, cinética de secagem, modelo matemático

\section{Mathematical modeling of drying process of fibrous mass of cassava}

\begin{abstract}
The purpose of this study was to develop a mathematical model to describe the drying process of cassava fibrous mass and compare it with other models from the literature. The Least-Squares Method is used to adjust the experimental values to a semi-empirical model. The experimental and predicted results were statisticaly compared using variance analysis to identify significant differences. The studied variables were temperature and air flow in tray dryer. The constructed model and Page model had values of mean relative error less than $6 \%$, and may be used to predict the drying kinetics.
\end{abstract}

Key words: Manihot esculenta Crantz, residue, by-product, drying kinetics, mathematical model 


\section{INTRODUÇÃO}

A mandioca (Manihot esculenta Crantz) é a quarta cultura mais importante para a alimentação do mundo e a principal nas regiões tropicais. Em 2009 a FAO divulgou que a raiz da mandioca e seus subprodutos são consumidos por mais de 800 milhões de pessoas. A produção mundial de mandioca é de aproximadamente 225,97 milhões de toneladas, sendo o Brasil o segundo maior produtor (Flôres, 2009).

A produção se destina ao consumo in natura e à industrialização, principalmente de produtos de relevância econômica, como a fécula e a farinha de mandioca; seu processamento resulta em uma variedade de resíduos sólidos, como as cascas, o bagaço e os descartes e os resíduos líquidos, como a manipueira e a água da lavagem da raiz. A quantidade de bagaço produzido em fecularias é bastante elevada, em média cerca de $900 \mathrm{~kg}$ para cada tonelada de raiz processada (Leonel et al., 1999). A composição desse resíduo pode variar dependendo da origem da mandioca e da técnica de processamento mas predominam carboidratos e fibras, com pequena quantidade de proteínas e lipídios (Pandey et al., 2000).

Os subprodutos da mandioca podem, quando lançados no ambiente, causar problemas sérios de poluição visto que apresentam, além da sua elevada carga orgânica, um composto passível de gerar cianeto, composto tóxico para a maioria dos seres de respiração aeróbia. Com isto, alternativas, como a utilização desses subprodutos na alimentação animal, adubação e biofertilizantes, vêm sendo propostas com o objetivo de minimizar este impacto ambiental negativo. Devido à grande quantidade de água utilizada durante o processamento da mandioca seus subprodutos apresentam umidade bastante elevada, sendo necessária a realização do processo de secagem para que se possa atingir maiores valores de matéria seca. A secagem pode ser realizada artificialmente com o auxílio de estufas, ou naturalmente, por exposição ao sol. Esta prática se faz necessária por facilitar o armazenamento e a conservação do material (Meneghetti \& Domingues, 2008). Este processo é vastamente utilizado para garantir maior estabilidade para alimentos visto que ele consiste na remoção da água contida no produto a determinado nível originando condições desfavoráveis à atividade biológica e às reações químicas e físicas que podem ocorrer durante o armazenamento (Martinazzo et al., 2007a).

De modo geral, os produtos biológicos são muito diferentes entre si devido a variações em sua forma, estrutura e dimensões, além de as condições de secagem serem muito diversas de acordo com as propriedades do ar utilizado e com a forma com que se faz o contato ar-produto. Quando o produto é colocado em contato com o ar de secagem ocorre transferência do calor do ar ao produto sob o efeito da diferença de temperatura existente entre eles. Simultaneamente, a diferença de pressão parcial de vapor de água existente entre o ar e a superfície do produto determina a transferência de vapor para o ar, uma parte do calor que chega ao produto é utilizada para evaporar a água e a outra para elevar sua temperatura.

As informações que as curvas de secagem podem fornecer estão relacionadas ao desenvolvimento do processo de secagem e ao dimensionamento do equipamento. O tempo de secagem referente a uma massa de produtos e o gasto energético, são algumas informações que se pode estimar através desses dados (Vilela \& Artur, 2008).

A modelagem matemática de um sistema dinâmico pode ser definida como um conjunto de equações que podem predizer a precisão do processo. Os modelos matemáticos podem ser bem diferentes dependendo do sistema considerado e das circunstâncias particulares de cada um deles e é em função disto que um modelo pode ser mais adequado do que outros (Ogata, 2003). Estudos sobre sistemas de secagem e sua otimização podem ser feitos por simulação matemática. $\mathrm{Na}$ simulação utiliza-se um modelo matemático que representa satisfatoriamente a perda de umidade do produto durante o período de secagem. Na literatura se encontram vários métodos teóricos, semiteóricos e empíricos para analisar a secagem de produtos (Afonso Júnior \& Corrêa, 1999; Martinazzo et al., 2007b; Ferreira \& Pena 2010; Resende et al., 2010).

Considerando a importância do estudo do processo de secagem e a escassez de informações teóricas a respeito dos fenômenos que ocorrem durante a secagem dos resíduos agroindustriais, o presente trabalho teve por objetivo elaborar um modelo matemático para predizer os resultados de umidade das amostras de massa fibrosa de mandioca durante o processo de secagem, em secador de bandeja usando-se como variáveis a temperatura e a vazão de ar.

\section{Material e Métodos}

Utilizou-se, como matéria-prima, a massa fibrosa da mandioca IAC-12, fornecida pela Fecularia Bela Vista - Febela Ltda., situada no município de Bela Vista de Goiás, GO, Brasil. As amostras foram coletadas na saída da centrífuga utilizandose materiais devidamente higienizados e esterilizados em autoclave. Após a coleta as amostras foram acondicionadas em sacos de polietileno de baixa densidade (PEBD), colocadas em caixa térmica com gelo artificial visando à manutenção das amostras durante o transporte até o laboratório de processamento de vegetais.

A massa fibrosa da mandioca foi caracterizada em relação aos teores de umidade, cinzas, extrato etéreo, proteínas e amido. Cinco amostras foram avaliadas. $\mathrm{O}$ teor de umidade foi determinado em estufa de ar a $105{ }^{\circ} \mathrm{C}$; o de cinzas por carbonização da matéria orgânica em forno mufla, o de extrato etéreo por extração em Soxhlet durante $10 \mathrm{~h}$ e posterior evaporação do solvente, o de proteínas pela técnica micro Kjeldahl, baseado em hidrólise e posterior destilação da amostra, todos de acordo com os métodos recomendados pela AOAC (1997). Todas as análises foram realizadas em triplicata.

$\mathrm{O}$ ensaio de secagem foi conduzido em secador de bandeja ( $1,90 \mathrm{~m}$ de altura por $0,80 \mathrm{~m}$ de largura, com capacidade de cinco bandejas metálicas de $55 \times 57 \mathrm{~cm}$ cada uma) com ventilação forçada utilizando-se, como variáveis independentes, a temperatura $\left(52,9-67,1^{\circ} \mathrm{C}\right)$ e o fluxo do ar $\left(0,019-0,023 \mathrm{~m}^{3} \mathrm{~kg}^{-1} \mathrm{~s}^{-1}\right)$. Para isto foi proposto um delineamento composto central rotacional $2^{2}$, com triplicata no ponto central, totalizando 11 experimentos (Barros Neto et al., 2010). Para avaliação dos resultados do processo de secagem amostras com $680 \mathrm{~g}$ de matéria-prima foram mantidas nas condições pre-estabelecidas 
pelo delineamento. O tempo de secagem necessário para a matéria-prima alcançar $9,0 \%$ foi determinado a partir do ajuste polinomial dos resultados com o intuito de minimizar os erros.

Testes preliminares foram realizados utilizando-se diferentes funções matemáticas, para obtenção de modelos que melhor representassem os valores experimentais. O grau de ajuste das funções foi avaliado por meio dos coeficientes de determinação. Os coeficientes numéricos das equações foram reparametrizados em função de diferentes modelos e calculados numericamente pelo método de mínimos quadrados. Os resultados experimentais e preditos foram comparados estatisticamente por análise de variância (Anova).

Optou-se, então, por utilizar ajustes polinomiais de segunda ordem de forma que os resultados acompanhassem a tendência dos valores experimentais, conforme descrito na Eq. 1.

$$
\mathrm{U}=\mathrm{C} 1+\mathrm{C} 2 \cdot \mathrm{t}+\mathrm{C} 3 \cdot \mathrm{t}^{2}
$$

em que:

U - porcentagem de umidade em base úmida do bagaço de mandioca

$$
\text { C1, C2 e C3 - coeficientes da Eq. } 1
$$

No ajuste polinomial foram calculados os coeficientes $\mathrm{C} 1$, C2 e C3 para cada curva, os quais foram ajustados a uma função linear (Eqs. 2, 3 e 4) dependente de outra função, denominada função Z (Eq. 5).

$$
\begin{aligned}
& \mathrm{C} 1=\mathrm{B} 1+\mathrm{B} 2 . \mathrm{Z} \\
& \mathrm{C} 2=\mathrm{B} 3+\mathrm{B} 4 . \mathrm{Z} \\
& \mathrm{C} 3=\mathrm{B} 5+\mathrm{B} 6 . \mathrm{Z}
\end{aligned}
$$

na qual:

B1, B3 e B5: B $_{i}$ e B2, B4 e B6: $B_{j}$

$$
\mathrm{Z}=\sin \left[\frac{\mathrm{a} 1}{\mathrm{a} 2 \cdot \mathrm{T} \cdot \mathrm{k} \cdot\left(\mathrm{F}_{\mathrm{ar}} \cdot \mathrm{D}_{\mathrm{ef}}\right)}\right]
$$

Para obtenção da função $\mathrm{Z}$ parametrizada em relação ao fluxo de ar $\left(\mathrm{F}_{\mathrm{ar}}, \mathrm{m}^{3} \mathrm{~kg}^{-1} \mathrm{~s}^{-1}\right)$ fornecido durante a secagem, os parâmetros a1 e a2 foram variados entre os valores 0,01 e 5 . Também foi estruturado um modelo estatístico de predição dos resultados de umidade da massa fibrosa de mandioca, construído a partir do delineamento experimental em que foram feitos, inicialmente, cinco modelos, fixando os tempos de coleta de amostra $(1,0 ; 1,5 ; 2,0 ; 2,5 ; 3,0 ; 4,0$ e 5,0 h) e se usando, como variáveis independentes, a temperatura e o fluxo de ar e, como variável dependente, a umidade da matéria-prima, após o tempo de secagem já mencionado. A partir desses modelos determinou-se a umidade para cada uma das condições do delineamento experimental. Para avaliação do modelo de secagem tendo o tempo como variável dependente, optou-se por fixar a umidade em $9,0 \%$, garantindo que a atividade de água seja baixa o suficiente para o seu armazenamento em ambiente fresco e seco.
Os novos modelos, empírico e estatístico, foram posteriormente comparados com os modelos de Crank e Page. A teoria da migração de água por difusão, baseada na segunda lei de Fick, foi considerada no estudo da cinética de secagem. Tendo em conta que a massa fibrosa da mandioca é representada por uma placa, fez-se uso da proposta de Crank (1975) a fim de se realizar a modelagem da cinética de secagem.

Em algumas situações a teoria difusional não é adequada para ajustar o comportamento da cinética de secagem devido a interferências no efeito de resistência interna do material. Para essas situações pode-se aplicar modelos empíricos, como o de Page (Page, 1949) mostrado na Eq. 6.

$$
Y=\frac{\bar{X}-X_{e q}}{X_{0}-X_{e q}}=\exp \left(-G \cdot t^{j}\right)
$$

em que:

G, j - coeficientes do modelo de Page

$\mathrm{Y}$ - adimensional de umidade

$\mathrm{X}$ - conteúdo de umidade

$\mathrm{X}_{0} \quad$ - conteúdo de umidade inicial

Xeq - umidade de equilíbrio

$$
\frac{\bar{X}-X_{\text {eq }}}{X_{0}-X_{\text {eq }}}=A \cdot \exp (-\mathrm{kt})
$$

na qual:

$$
\mathrm{k}=\frac{\pi^{2} \cdot \mathrm{D}_{\mathrm{ef}}}{\mathrm{r}^{2}}
$$

Como a umidade de equilíbrio $\left(\mathrm{X}_{\mathrm{eq}}, \%\right)$ é função da umidade relativa de ar (UR, \%) e da temperatura e o processo de secagem se dá a condições variáveis de temperatura e umidade, deve-se calcular um $\mathrm{X}_{\text {eq }}$ para cada instante da secagem. Esses valores podem ser obtidos através do gráfico de umidade de equilíbrio versus umidade relativa, ou através das equações de HendersonThompson (Eqs. 9, 10 e 11).

$$
\mathrm{Xeq}=0,01(\mathrm{a} / \mathrm{b})^{0,409}
$$

na qual:

$$
\begin{gathered}
\mathrm{a}=-\mathrm{LN}(1-\mathrm{UR}) \\
\mathrm{b}=0,000019187(\mathrm{~T}+51,161) \\
\text { RESULTADOS E DisCUSSÃO }
\end{gathered}
$$

Os dados médios obtidos para umidade, proteína, extrato etéreo e cinzas da massa fibrosa da mandioca, foram de 89,10, $1,68,0,97$ e 1,85\%, respectivamente. Meneghetti \& Domingues (2008) mencionam, na sua discussão em relação ao farelo de bagaço de mandioca, que o subproduto apresentou, antes da secagem, cerca de $75 \%$ de umidade, $15,83 \%$ abaixo do verificado nesta pesquisa. Ramos et al. (2000) encontraram, 
em seu trabalho, $82,77 \%$ de umidade no bagaço da mandioca, valor 7,1\% menor que o verificado no presente trabalho. Em relação à proteína, dados semelhantes foram encontrados por Jasko et al. (2011) e Pandey et al. (2000). Já em relação ao estrato etéreo e às cinzas os valores encontrados pelos autores foram 0,1 e $2,0 \%$, respectivamente.

$\mathrm{Na}$ Tabela 1 são mostrados os resultados do delineamento experimental utilizado no processo de secagem da massa fibrosa de mandioca assim como os valores de temperatura e fluxo de ar.

Tabela 1. Resultados do delineamento experimental composto central rotacional $2^{2}$ para o processo de secagem do bagaço de mandioca até umidade de 9,0\%

\begin{tabular}{cccc}
\hline Experimento & $\begin{array}{c}\mathbf{T}_{\text {ar }} \\
\left({ }^{\circ} \mathbf{C}\right)\end{array}$ & $\begin{array}{c}\mathbf{F}_{\text {ar }} \\
\left(\mathbf{m}^{\mathbf{k}} \mathbf{k g ~ s}\right.\end{array}$ & $\begin{array}{c}\text { Tempo } \\
(\mathbf{h})\end{array}$ \\
1 & 55 & 0,016 & 9,50 \\
2 & 65 & 0,016 & 6,02 \\
3 & 55 & 0,022 & 6,64 \\
4 & 65 & 0,022 & 4,36 \\
5 & 60 & 0,019 & 5,92 \\
6 & 60 & 0,019 & 6,13 \\
7 & 60 & 0,019 & 5,78 \\
8 & 53 & 0,019 & 6,75 \\
9 & 60 & 0,023 & 4,66 \\
10 & 67 & 0,019 & 4,77 \\
11 & 60 & 0,015 & 5,97 \\
\hline
\end{tabular}

O comportamento cinético apresentou tendência semelhante nos diferentes experimentos de secagem da massa fibrosa da mandioca porém com intensidades diferentes. Kurozawa (2005) observou, em experimentos de secagem usando temperaturas de 45 a $75{ }^{\circ} \mathrm{C}$ e velocidade do ar entre 1,2 e $2,3 \mathrm{~m} \mathrm{~s}^{-1}$, comportamento cinético parecido com os do presente trabalho, mas utilizando cogumelo Agaricusblazei como matéria-prima. A partir dos resultados da análise de variância verificaram-se efeitos lineares significativos das variáveis temperatura $(\mathrm{p}=$ $0,0006)$ e fluxo de $\operatorname{ar}(p=0,0052)$ sobre o tempo de secagem A Eq. 12 representa o modelo ajustado para o tempo de secagem pois foram excluídos os termos não significativos a $5 \%$ de probabilidade.

$$
\mathrm{t}=5,55-1,73 \mathrm{~T}_{\mathrm{ar}}-1,21 \mathrm{~F}_{\mathrm{ar}}
$$

em que:

$$
\begin{array}{ll}
\mathrm{t} & \text { - tempo de secagem, } \mathrm{h} \\
\mathrm{T}_{\mathrm{ar}} & \text { - temperatura do ar, }{ }^{\circ} \mathrm{C} \\
\mathrm{F}_{\mathrm{ar}} & \text { - fluxo de ar, } \mathrm{m}^{3} \mathrm{~kg} \mathrm{~s}^{-1}
\end{array}
$$

O coeficiente de determinação ajustado $\left(\mathrm{R}^{2}\right)$ obtido para o modelo ajustado para tempo de secagem, explicou $81 \%$ do total das respostas. $\mathrm{O}$ modelo ajustado prediz o tempo necessário para alcançar a umidade final estudada ( $9,0 \%$ em base úmida) quando se utilizam a temperatura e o fluxo de ar, dentro das faixas estudadas no delineamento experimental. A variação entre os coeficientes de determinação do modelo completo e do modelo ajustado (sem os parâmetros estatisticamente não significativos) não foi grande; mesmo assim, optou-se por utilizar o segundo por este apresentar melhor ajuste. Tendo em vista que o desvio-padrão para os experimentos nos pontos centrais de temperatura e vazão de ar foi pequeno, pode-se comprovar a repetibilidade do processo.

Na Tabela 2 é apresentada a análise de variância (Anova) para o delineamento experimental no qual todos os parâmetros foram utilizados. O modelo foi significativo (p-valor 0,001 ) uma vez que o $\mathrm{F}$ calculado para a regressão é 5,06 vezes maior que $\mathrm{F}$ tabelado.

Tabela 2. Análise de variância do delineamento experimental composto central rotacional $2^{2}$ para o processo de secagem do bagaço de mandioca

\begin{tabular}{lccccc}
\hline $\begin{array}{l}\text { Fonte de } \\
\text { variação }\end{array}$ & $\begin{array}{c}\text { Soma dos } \\
\text { quadrados }\end{array}$ & $\begin{array}{c}\text { Grau de } \\
\text { liberdade }\end{array}$ & $\begin{array}{c}\text { Quadrado } \\
\text { médio }\end{array}$ & $\mathbf{F}_{\text {calculado }}$ & p-valor \\
Regressão & 35,852 & 2 & 17,925 & 15,224 & 0,001 \\
Resíduos & 10,597 & 9 & 1,177 & & \\
Total & 46,449 & 10 & & & \\
\hline Porcentagem de variação explicada $\left(\mathrm{R}^{2}\right)=95 \% ; \mathrm{F}_{2 ;: 0,1}=3,01$ & &
\end{tabular}

Nas condições de $67^{\circ} \mathrm{C}$ e $0,023 \mathrm{~m}^{3} \mathrm{~kg}^{-1} \mathrm{~s}^{-1}$ verificou-se a maior perda de umidade da massa fibrosa de mandioca em menor tempo. Kurozawa (2005) investigando a secagem de cogumelo com delineamento experimental idêntico ao deste trabalho porém com faixas de temperatura entre 40 e $80^{\circ} \mathrm{C}$ e velocidade do ar entre 1,0 e $2,5 \mathrm{~m} \mathrm{~s}^{-1}$, verificou o mesmo comportamento, com efeito significativo linear das variáveis de estudo. Observou-se, nos resultados, que os efeitos lineares da temperatura e do fluxo de ar apresentam um efeito negativo para o tempo de secagem. $\mathrm{O}$ aumento da temperatura ou do fluxo de ar acarretou diminuição do tempo de secagem o que é interessante economicamente quando se busca operacionalizar um processo como este.

A partir dos resultados do delineamento experimental composto central rotacional $2^{2}$ foi desenvolvido um modelo empírico baseado no ajuste da cinética do processo de secagem. O ajuste polinomial foi aquele que melhor se aproximou dos valores experimentais. Polinômios de graus elevados também foram testados mas apesar de terem apresentado altos coeficientes de determinação, observou-se instabilidade dos modelos, no que se refere aos resultados preditos impossibilitando sua confiabilidade. Os valores de $\mathrm{R}^{2}$ encontrados permaneceram acima de 0,997 e podem ser observados juntamente com os coeficientes do polinômio, na Tabela 3. Para obtenção do modelo o conjunto de valores de cada coeficiente ( $\mathrm{C} 1, \mathrm{C} 2$ e $\mathrm{C} 3)$ apresentado na Tabela 3, foi ajustado a uma função linear (Eqs. 2, 3 e 4).

Assim, o par (a1, a2) que apresentou o maior valor para o coeficiente de determinação foi escolhido.

Na Tabela 4 são mostrados os coeficientes $B_{i}$ e $B_{j}$, referente às Eq. 2, 3 e 4, juntamente com os parâmetros a1 e a2 da função parametrizada $Z$ (Eq. 5). Os valores dos coeficientes de determinação para função linear parametrizada foram 0,8709 , 0,7936 e 0,8198 , sinalizando bom ajuste à função trigonométrica utilizada.

Na Figura 1 são mostradas as curvas do modelo nas condições 1,2 e 3 do delineamento experimental composto central rotacional.

Pelo comportamento das curvas pode-se verificar, também, a influência das variáveis de processo durante a secagem da massa fibrosa de mandioca. Com o modelo desenvolvido é 
Tabela 3. Coeficientes de determinação $\left(R^{2}\right)$ e coeficientes $\mathrm{C} 1, \mathrm{C} 2$ e $\mathrm{C} 3$, referentes à Eq. 1, obtidos através do ajuste polinomial dos resultados experimentais do processo de secagem do bagaço de mandioca

\begin{tabular}{|c|c|c|c|c|c|}
\hline \multirow{2}{*}{$\begin{array}{l}\text { Temperatura } \\
\left({ }^{\circ} \mathrm{C}\right)\end{array}$} & \multirow{2}{*}{$\begin{array}{c}\text { Fluxo de ar } \\
\left(\mathrm{m}_{\text {ar }}^{3} \mathrm{~kg}_{\text {amostra }} \mathrm{S}^{-1}\right)\end{array}$} & \multicolumn{3}{|c|}{ Constantes do polinômio } & \multirow{2}{*}{$\mathbf{R}^{2}$} \\
\hline & & C1 & C2 & C3 & \\
\hline 55,0 & 0,016 & 68,38 & $-12,69$ & 0,66 & 0,998 \\
\hline 65,0 & & & $-16,72$ & 1,11 & 999 \\
\hline & & & & & 999 \\
\hline 65,0 & & & $-19,64$ & & 0,999 \\
\hline 60,0 & & & -17 & 1, & 0,9 \\
\hline & & & $-13,60$ & 0,63 & 0,999 \\
\hline & & & & & 0,999 \\
\hline 9 & & & -13 & 0,6 & 0,999 \\
\hline & & & -17 & 0, & 0,999 \\
\hline & & & & & 0,9 \\
\hline 60,0 & 0,015 & 69,73 & $-14,05$ & 0,62 & 0,999 \\
\hline
\end{tabular}

Tabela 4. Coeficiente $\left(B_{i}\right.$ e $\left.B_{j}\right)$ e parâmetros (a1 e a2) usados nas Eqs. 2, 3, 4 e 5

\begin{tabular}{crrrcc}
\hline Coeficientes & \multicolumn{1}{c}{$\mathbf{B}_{\mathbf{i}}$} & $\mathbf{B}_{\mathbf{i}}$ & $\mathbf{a} 1$ & $\mathbf{a} 2$ & $\mathbf{R}^{\mathbf{2}}$ \\
C1 & 69,125 & $-2,320$ & 4,82 & 1,51 & 0,870 \\
C2 & $-16,067$ & $-2,597$ & 4,98 & 1,01 & 0,793 \\
C3 & 1,000 & 0,395 & 4,94 & 1,38 & 0,819 \\
\hline
\end{tabular}

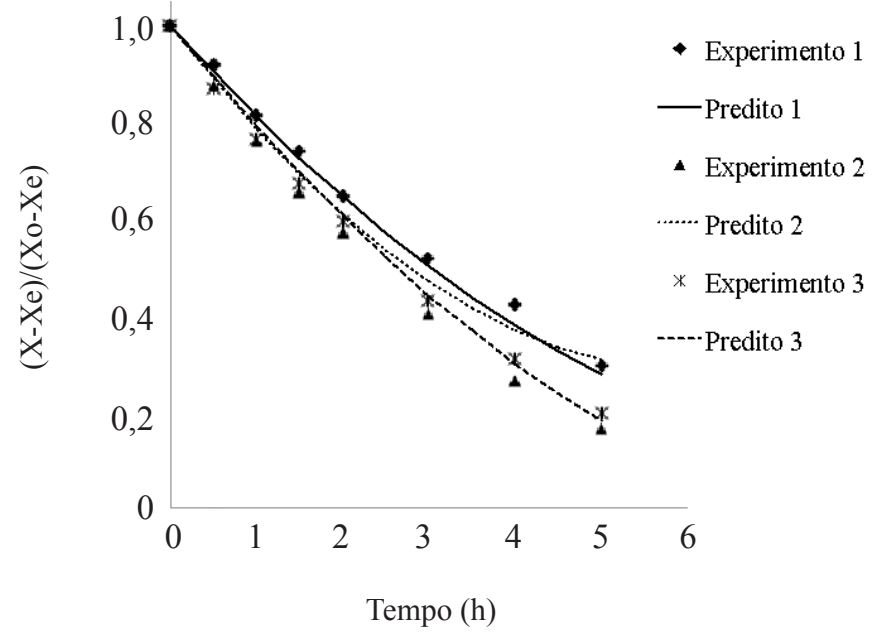

Figura 1. Umidades experimentais (pontos) e preditas pelo modelo (linhas contínua) do processo de secagem do bagaço de mandioca para as condições experimentas $1\left(55^{\circ} \mathrm{Ce} 0,016 \mathrm{~m}_{\text {ar }}^{3} \mathrm{~kg}_{\text {amostra }} \mathrm{s}^{-1}\right), 2\left(65^{\circ} \mathrm{C}\right.$ e $\left.0,016 \mathrm{~m}^{3}{ }_{\text {ar }} \mathrm{kg}_{\text {amostra }} \mathrm{s}^{-1}\right)$ e $3\left(55^{\circ} \mathrm{C}\right.$ e $0,022 \mathrm{~m}^{3}{ }_{\text {ar }} \mathrm{kg}_{\text {amostra }}$ $\left.\mathrm{s}^{-1}\right)$ do delineamento composto central rotacional

possível calcular o tempo necessário para alcançar umidade nas condições estabelecidas, desde que esteja dentro da faixa estudada. Assim como os demais modelos não se pode garantir resultados precisos de umidade nas faixas que extrapolam os limites de temperatura e fluxo de ar estudados.

O efeito exercido pela temperatura no processo de secagem se deve à energia cinética, associada às moléculas de água presentes no material (Shivhare et al., 2004). Com o aumento da temperatura se eleva a energia cinética do sistema e com isto ocorre diminuição das forças de atração entre as moléculas de água e os demais constituintes que compõem o material, conduzindo a um decréscimo da umidade. Vários pesquisadores observaram este comportamento em diferentes alimentos (Park et al., 2001; Viswanathan et al., 2003; Shivhare et al., 2004; Vilhalva et al., 2012). Doymaz \& Ismail (2011) demonstraram, durante estudos de secagem, que a temperatura influencia de forma muito significativa no processo, pelo consequente aumento da difusividade.

Pode-se notar que os ensaios que apresentaram maior variação nos valores foram os experimentos 1 e 4 que são justamente os níveis inferiores e superiores, respectivamente, para temperatura e fluxo de ar. Na Figura 2 são mostradas as curvas de secagem nas condições do delineamento experimental. Independentemente dos trabalhos sobre secagem, Crank (1975) calculou grande número de soluções da equação de difusão para condições iniciais e de contorno variadas; entretanto, essas soluções se aplicavam aos sólidos de formas geométricas simples (corpos semi-infinitos, placas, cilindros e esferas) e quando a difusividade era constante ou variava linearmente ou exponencialmente com a concentração de água.

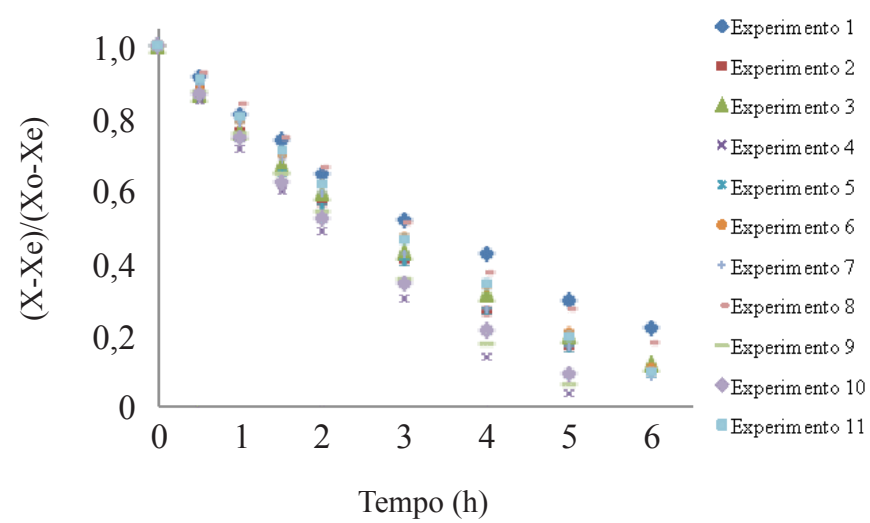

Figura 2. Resultados de adimensional de umidade para o processo de secagem da massa fibrosa de mandioca a partir do delineamento experimental

Fazendo a análise exponencial dos resultados obtém-se um modelo matemático (Eq. 11). A partir desta equação foi possível calcular os resultados de constante de equilíbrio $(\mathrm{k})$ para os modelos de Crank e Page (Tabela 5). Também são mostrados os resultados de difusividade efetiva $\left(\mathrm{D}_{\mathrm{ef}}\right)$ para os dois modelos.

Quanto menos drástica foi a condição de secagem, menor $\mathrm{k}$ (constante de secagem) foi observado. Esta afirmação mostra o quanto os resultados foram coerentes frente a uma análise. O mesmo comportamento se observou quando foram analisados os resultados de difusividade efetiva. Analisando tal comportamento pode-se afirmar que quanto maior a temperatura de processo mais acentuado é o processo de secagem, isto é, quando se comparam as amostras secadas a uma temperatura maior com aquelas secadas em uma temperatura menor, observa-se que, para que as últimas alcancem o mesmo valor de umidade das anteriores, faz-se necessário maior tempo de processo. Aghbashlo et al. (2009) encontraram valores de $\mathrm{D}_{\text {ef }}$ parecidos que o presente trabalho (variando de $3,17 \times 10^{-7} \mathrm{a}$ $15,45 \times 10^{-7} \mathrm{~m}^{2} \mathrm{~s}^{-1}$ ). Referidos pesquisadores trabalharam com a secagem de batata com umidade inicial de $362 \mathrm{~g}(100 \mathrm{~g})^{-1}$ (b.s.), teor mais de três vezes superior à da polpa residual de 
Tabela 5. Constante de equilíbrio $\left(\mathrm{h}^{-1}\right)$ e difusividade efetiva $\left(\mathrm{m}^{2} \mathrm{~s}^{-1}\right)$ para cada experimento de secagem de bagaço de mandioca

\begin{tabular}{ccccc}
\hline Experimento & $\begin{array}{c}\mathbf{K} \\
\text { (Page) }\end{array}$ & $\begin{array}{c}\mathbf{K} \\
\text { (Crank) }\end{array}$ & $\begin{array}{c}\mathbf{D}_{\text {ef }} \\
\text { (Page) }\end{array}$ & $\begin{array}{c}\mathbf{D}_{\text {ef }} \\
\text { (Crank) }\end{array}$ \\
1 & 0,195340 & 0,097581 & $7,03223 \mathrm{E}-08$ & $3,51290 \mathrm{E}-08$ \\
2 & 0,260269 & 0,158064 & $9,36969 \mathrm{E}-08$ & $5,69031 \mathrm{E}-08$ \\
3 & 0,252386 & 0,143226 & $9,08591 \mathrm{E}-08$ & $5,15613 \mathrm{E}-08$ \\
4 & 0,312889 & 0,208564 & $1,12640 \mathrm{E}-07$ & $7,50832 \mathrm{E}-08$ \\
5 & 0,273263 & 0,163774 & $9,83745 \mathrm{E}-08$ & $5,89588 \mathrm{E}-08$ \\
6 & 0,213723 & 0,130945 & $7,69402 \mathrm{E}-08$ & $4,71402 \mathrm{E}-08$ \\
7 & 0,222338 & 0,149784 & $8,00419 \mathrm{E}-08$ & $5,39222 \mathrm{E}-08$ \\
8 & 0,168341 & 0,118003 & $6,06026 \mathrm{E}-08$ & $4,24812 \mathrm{E}-08$ \\
9 & 0,257506 & 0,176101 & $9,27021 \mathrm{E}-08$ & $6,33963 \mathrm{E}-08$ \\
10 & 0,285633 & 0,174307 & $1,02828 \mathrm{E}-07$ & $6,27506 \mathrm{E}-08$ \\
11 & 0,196849 & 0,131184 & $7,08658 \mathrm{E}-08$ & $4,72263 \mathrm{E}-08$ \\
\hline
\end{tabular}

batata. Neste trabalho foi utilizado secador contínuo com ar em contracorrente. Os resultados encontrados mostraram uma boa eficiência do processo quando comparado com os sistemas utilizados por esses pesquisadores mencionados. Tal observação é de fundamental importância visto que sistema de secagem é um dos requisitos que interferem significativamente na $\mathrm{D}_{\text {ef }}$, uma vez que essa grandeza não é intrínseca ao material mas sim das condições de secagem (Oliveira et al., 2006).

$\mathrm{Na}$ Tabela 6 são mostrados os valores experimentais e preditos e os erros associados aos modelos avaliados neste trabalho.

Devido os resultados serem parecidos, optou-se por ilustrar somente os resultados do experimento nas condições de $55^{\circ} \mathrm{C}$ e $0,016 \mathrm{~m}^{3} \mathrm{~kg}^{-1} \mathrm{~s}^{-1}$ para facilitar a visualização. Os resultados preditos pelos modelos de Page e o elaborado neste trabalho, mostram uma aproximação grande dos resultados experimentais. Devido a pequenas variações no final do processo de secagem resultarem em um erro relativamente alto, se explica o comportamento da secagem no modelo elaborado nesta fase. Tal variação foi observada nos dois últimos pontos de coleta de amostra sendo que nas coletas anteriores o modelo elaborado apresentou alguns ajustes melhores que o do modelo de Page. De modo geral, dentre os quatro modelos analisados observou-se um ajuste melhor do modelo de Page seguido do modelo elaborado. Nos dois modelos os valores de erro relativo médio foram inferiores a $6 \%$, podendo ser utilizados para a predição da cinética de secagem. Resultados semelhantes foram obtidos por Simal et al. (2005) para a secagem de kiwi em cubos a temperatura variando de 30 a $90{ }^{\circ} \mathrm{C}$, ao ajustarem os dados experimentais aos modelos. Panchariya et al. (2002) observaram que o modelo exponencial foi o que melhor descreveu a cinética de secagem de chá preto (temperatura de $80-120^{\circ} \mathrm{C}$ e velocidade do ar de $0,25-0,65 \mathrm{~m} \mathrm{~s}^{-1}$ ). Barbosa et al. (2007) fixando velocidade do ar em $0,29 \mathrm{~m} \mathrm{~s}^{-1}$ e variando a temperatura do ar de secagem $\left(40-80{ }^{\circ} \mathrm{C}\right) \mathrm{em}$ folhas de erva cidreira, observaram que o modelo de Page foi um dos que melhor se ajustaram. Na Figura 3 são mostradas as diferenças entre os modelos estatístico, de Crank, de Page e do modelo desenvolvido neste trabalho. Para facilitar a visualização foi escolhido o experimento 1 do delineamento experimental para construção do gráfico de Andrews.

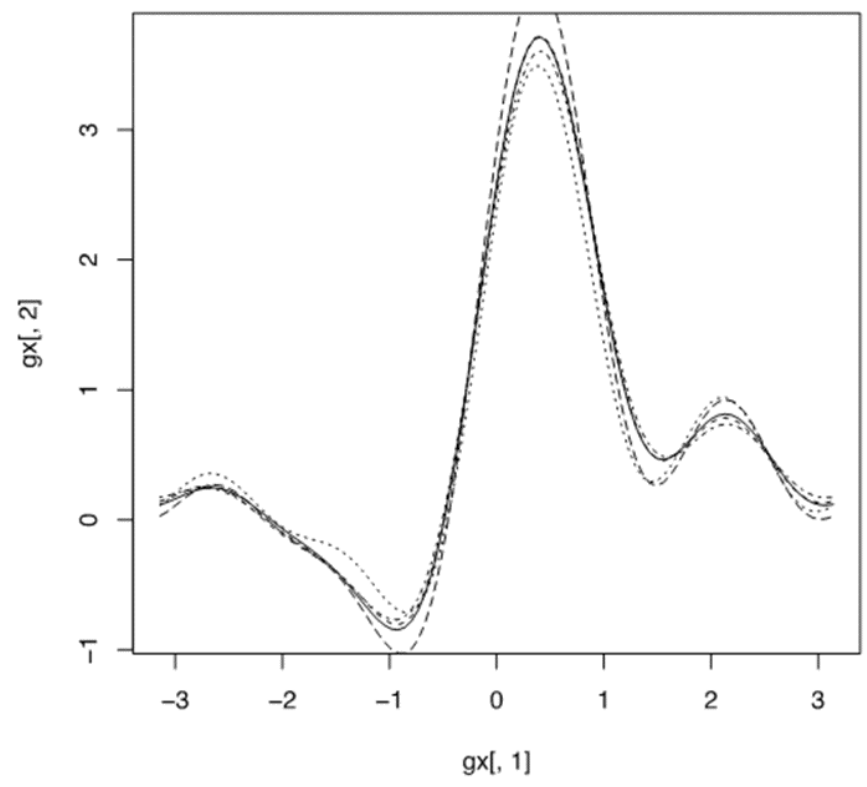

Figura 3. Diferença entre os modelos de Page (-), Crank ( - ), desenvolvido no presente trabalho ( - ) e estatístico (-... ) referentes aos experimentos 1 do delineamento experimental composto central rotacional $2^{2}$

Avaliando a distância euclidiana, assim como a análise de variância dos resultados, observa-se diferença significativa entre os resultados dos modelos sendo aconselhável avaliar as diferenças pelos resultados apresentados na Tabela $6 . \mathrm{Na}$ Figura 4 se encontram os resultados experimentais e preditos para os modelos estatísticos, Page, Crank e o desenvolvido no presente trabalho.

Estudando a modelagem do processo de secagem, muitos pesquisadores observaram que o modelo de Page se ajusta satisfatoriamente aos resultados experimentais dos mais

Tabela 6. Valores experimentais e preditos do adimensional de umidade para os diferentes modelos matemáticos utilizados na cinética de secagem da massa fibrosa de mandioca $55^{\circ} \mathrm{C}$ e $0,016 \mathrm{~m}_{\text {ar }}^{3} \mathrm{~kg}_{\text {amostra }} \mathrm{s}^{-1}$

\begin{tabular}{|c|c|c|c|c|c|c|c|c|c|}
\hline \multirow{2}{*}{ Tempo (h) } & \multirow{2}{*}{ Obs } & \multicolumn{2}{|c|}{ Page } & \multicolumn{2}{|c|}{ Crank } & \multicolumn{2}{|c|}{ Modelo } & \multicolumn{2}{|c|}{ Estatístico } \\
\hline & & Pred. & $\mathbf{P}(\%)$ & Pred. & $\mathbf{P}(\%)$ & Pred. & $\mathbf{P}(\%)$ & Pred. & $\mathbf{P}(\%)$ \\
\hline 0,5 & 0,916 & 0,914 & 0,24 & 0,776 & 15,24 & 0,900 & 1,76 & 0,953 & 4,07 \\
\hline 1,5 & 0,737 & 0,734 & 0,41 & 0,632 & 14,30 & 0,718 & 2,64 & 0,810 & 9,92 \\
\hline 2,0 & 0,644 & 0,653 & 1,31 & 0,583 & 9,50 & 0,636 & 1,31 & 0,736 & 14,33 \\
\hline 3,0 & 0,515 & 0,510 & 0,94 & 0,506 & 1,78 & 0,487 & 5,29 & 0,626 & 21,69 \\
\hline
\end{tabular}

Obs - adimensional de umidade experimental; Pred. - Valores preditos; $P$ - erro 


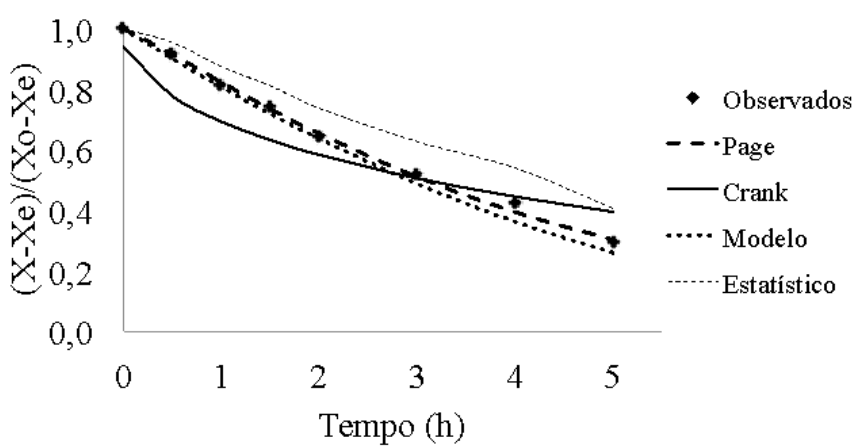

Figura 4. Valores experimentais e preditos para os modelos matemáticos utilizados na cinética de secagem da massa fibrosa de mandioca na condição de $55^{\circ} \mathrm{C}$ e $0,016 \mathrm{~m}_{\text {ar }}^{3} \mathrm{~kg}_{\text {amostra }} \mathrm{s}^{-1}$

diferentes tipos de matérias-primas (Demir et al., 2004; Bozkir, 2006; Aghbashlo et al., 2009; Doymaz \& Ismail, 2011). Tal constatação se mostra bastante interessante uma vez que a diversidade de produtos que sofre o processo de secagem é bastante elevada. Alternativamente, o presente trabalho vem enriquecer os estudos de modelagem do processo cinético de secagem da massa fibrosa de mandioca visto que o uso deste modelo matemático desenvolvido pode ser capaz de predizer resultados do tempo de secagem de forma precisa, como outros modelos da literatura.

\section{Conclusões}

1. O modelo construído apresenta boa aproximação dos resultados experimentais, porém o modelo de Page foi o que melhor se ajustou até o limite de umidade final estabelecido ( $9 \%$ base úmida).

2. O modelo construído e o de Page apresentaram valores de erro relativo médio inferiores a $6 \%$, podendo ser utilizado para predição da cinética de secagem.

3. O novo modelo desenvolvido agrega informações para a construção de modelos matemáticos na predição de resultados em processos de secagem.

\section{Literatura Citada}

Afonso Júnior, P. C.; Corrêa, P. C. Comparação de modelos matemáticos para descricão da cinética de secagem em camada fina de sementes de feijão. Revista Brasileira de Engenharia Agrícola e Ambiental, v.3, p.349-353, 1999.

Aghbashlo, M.; Kianmehr, M. H.; Arabhosseini, A. Modeling of thin-layer drying of potatos lices in length of continuous band dryer. Energy Conversion and Management, v.50, p.1348-1355, 2009.

AOAC - Official methods of analysis of AOAC International, 16. 3.ed. Maryland: AOAC International. 1997.

Barbosa, F. F.; Melo, E. C.; Santos, R. H. S.; Rocha, R. P.; Martinazzo, A. P.; Radünz, L. L.; Gracia, L. M. N. Evaluation of mathematical models for prediction of thinlayer drying of Brazilia lemon-scented verbena leaves (Lippia alba (Mill) N.E. Brown). Revista Brasileira de Produtos Agroindustriais, v.9, p.73-82, 2007.
Barros Neto, B. B.; Scarmínio, I. S.; Bruns, R. E. Como fazer experimentos: Pesquisa e desenvolvimento na ciência e na indústria. 4.ed. Campinas: UNICAMP, 2010, 404p.

Bozkir, O. Thin-layer drying and mathematical modelling for washed dry apricots. Journal of Food Engineering, v.77, p.146-151, 2006.

Crank, J. The mathematics of diffusion. 2.ed. Oxford: Claredon Press, 1975, 414p.

Demir, V.; Gunhan, T.; Yagcioglu, A. K.; Degirmencioglu, A. Mathematical modelling and the determination of some quality parameters of air-dried bay leaves. Biosystems Engineering, v.88, p.325-335, 2004.

Doymaz, I.; Ismail, O. Drying characteristics of sweet cherry. Food and Bioproducts Processing, v.89, p.31-38, 2011.

Ferreira, M. F. P.; Pena, R. S. Estudo da secagem da casca do maracujá amarelo. Revista Brasileira de Produtos Agroindustriais, v.12, p.15-28, 2010.

Flôres, D. Caracterização molecular e análise filogenética do fitoplasma agente do superbrotamento da mandioca (Manihot esculenta) no estado de São Paulo. Piracicaba: USP, 2009. 75p. Dissertação Mestrado

Jasko, A. C.; Andrade, A.; Campos, P. F.; Padilha, L.; Paul, R. B.; Quast, L. B.; Schnitzler, E.; Demiate, I. M. Caracterização físico-química de bagaço de mandioca in natura e após tratamento hidrolítico. Revista Brasileira de Tecnologia Agroindustrial, v.5, p.427-441, 2011.

Kurozawa, L. E. Efeito das condições de processo na cinética de secagem de cogumelo (Agaricusblazei). Campinas: UNICAMP, 2005. 141p. Dissertação Mestrado

Leonel, M.; Cereda, M. P.; Roau, X. Aproveitamento de resíduo da produção de etanol a partir de farelo de mandioca, como fonte de fibras dietéticas. Ciência e Tecnologia de Alimentos, v.19, p.241-245, 1999.

Martinazzo, A. P.; Corrêa, P. C.; Melo, E. C.; Barbosa, F. F. Difusividade efetiva em folhas de Cymbopogon citratus (DC.) Stapf submetidas à secagem com diferentes comprimentos de corte e temperaturas do ar. Revista Brasileira de Plantas Medicinais, v.9, p.68-72, 2007a.

Martinazzo, A. P.; Corrêa, P. C.; Resende, O.; Melo, E. C. Análise e descrição matemática da cinética de secagem de folhas de capim-limão. Revista Brasileira de Engenharia Agrícola e Ambiental, v.11, p.301-306, 2007b.

Meneghetti, C. D. C.; Domingues, J. L. Características nutricionais e uso de subprodutos da agroindústria na alimentação de bovinos. Revista Eletrônica Nutritime, v.5, p.512-536, 2008.

Ogata, K. Engenharia de controle moderno. 4.ed. São Paulo: Prentice Hall, 2003. 940p.

Oliveira, R. A.; Oliveira, W. P.; Park, K. J. Determinação da difusividade efetiva de raiz de chicória. Engenharia Agrícola, v.26, p.181-189, 2006.

Page, G. E. Factors influencing the maximum of air drying shelled corn in thin layer. 1949. Indiana: Purdue University, 1949. Thesis M.Sc.

Panchariya, P. C.; Popovic, D.; Sharma, A. L. Thin-layer modeling of black tea drying process. Journal of Food Engineering, v.52, p.349-357, 2002. 
Pandey, A.; Soccol, C. R.; Nigam, P.; Soccol, V. T.; Vandenbergue, L. P. S.; Mohan, R. Botechnological potencial of agro-industrial residue II: Cassava bagasse. Bioresource Technology, v.74, p.81-87, 2000.

Park, K. J.; Bin, A.; Brod, F. P. R. Obtenção das isotermas de sorção e modelagem matemática para a pêrabartlett (Pyrus sp.) com e sem desidratação osmótica. Ciência e Tecnologia de Alimentos, v.21, p.73-77, 2001.

Ramos, P. R.; Prates, E. R.; Fontanelli, R. S. Uso do bagaço de mandioca em substituição ao milho no concentrado para bovinos em crescimento. Digestibilidade aparente consumo de nutrientes digeríveis, ganho de peso e conversão alimentar. Revista da Sociedade Brasileira de Zootecnia, v.29, p.295-299, 2000.

Resende, O.; Ferreira, L. U.; Almeida, D. P. Modelagem matemática para descrição da cinética de secagem do feijão Adzuki (Vigna angularis). Revista Brasileira de Produtos Agroindustriais, v.12, p.171-178, 2010.
Shivhare, U. S.; Arora, S.; Ahmed, J.; Raghavan, G. S. V. Moisture adsorption isotherms for mushroom. Lebensmittel - Wissenschaftand Technologies, v.37, p.133-137, 2004.

Simal, S.; Femenia, A.; Garau, M. C.; Rosselló, C. Use of exponential, Page's and diffusional models to simulate the drying kinetics of kiwi fruit. Journal of Food Engineering, v.66, p.323-328, 2005.

Vilela, C. A. A.; Artur, P. O. Secagem do açafrão (Curcuma longa L.) em diferentes cortes geométricos. Ciência e Tecnologia de Alimentos, v.28, p.387-394, 2008.

Vilhalva, D. A. A.; Soares Júnior, M. S.; Caliari, M.; Silva, F. A. Secagem convencional de casca de mandioca proveniente de resíduos de indústria de amido. Pesquisa Agropecuária Tropical, v.42, p.331-339, 2012.

Viswanathan, R.; Jayas, D. S.; Hulasare, R. B. Sorption isotherms of tomatos lices and onion shreds. Biosystems Engineering, v.86, p.465-472, 2003. 